\title{
Influência da temperatura na taxa respiratória de abelhas forrageiras Trigona spinipes (Fabricius) (Hymenoptera,Apidae, Meliponinae) durante períodos de verão e inverno
}

\author{
Oilton J. D. Macieira \& Edson A. Proni
}

Departamento de Biologia Animal e Vegetal, Universidade Estadual de Londrina. Campus Universitário, Caixa Postal 6001, 860651-990 Londrina, Paraná, Brasil.E-mail: macieira@uel.br; proni@uel.br

\begin{abstract}
Influence of the temperature in the respiratory rate of bee workers of Trigona spinipes (Fabricius) (Hymenoptera, Apidae, Meliponinae) during periods of summer and winter. The results obtained on the influence of the temperature in the respiratory rate of workers of Trigona spinipes (Fabricius, 1793) showed an increase of the oxygen consumption in agreement with the increase of the temperatures. It was also possible to observe a more intense respiratory metabolism in the winter than in the summer, mainly in the temperatures between 30 and $35^{\circ} \mathrm{C}$. The results of the analysis of factorial variance, being compared summer and winter showed significant differences among $15 ; 20$ and $30^{\circ} \mathrm{C}$. Regarding the thermal preferences, it was observed in the winter and in the summer an interval between 20 and $30^{\circ} \mathrm{C}$ where the oxygen consumption stabilized showing a possible ideal thermal track for that bee species.
\end{abstract}

KEY WORDS. Oxygen consumption, respiratory metabolism, stingless bees.

RESUMO. Os resultados obtidos sobre a influência da temperatura na taxa respiratória de forrageiras de $T$. spinipes (Fabricius, 1793) mostraram um aumento no consumo de oxigênio de acordo com o aumento das temperaturas. Também foi possível observar um metabolismo respiratório mais intenso no inverno do que no verão, principalmente nas temperaturas entre 30 e $35^{\circ} \mathrm{C}$. Os resultados da análise de variância fatorial, comparando-se verão e inverno mostraram diferenças significativas entre as temperaturas $15 ; 20$ e $30^{\circ} \mathrm{C}$. Com relação às preferências térmicas observou-se tanto no inverno como no verão um intervalo entre 20 e $30^{\circ} \mathrm{C}$, sendo que o consumo de oxigênio estabilizou mostrando uma possível faixa térmica ideal para essa espécie de abelha. PALAVRAS CHAVE. Abelhas sem ferrão, consumo de oxigênio, metabolismo respiratório.

Trigona spinipes (Fabricius, 1793), também conhecida como irapuá, arapuá, arapuã, é uma espécie de abelha sem ferrão pertencente ao grupo dos Meliponíneos, encontrada no Brasil desde o Ceará até o Rio Grande do Sul, Paraguai e Província de Missiones (Argentina). Constroem ninhos externos envolvendo galhos de árvores e são consideradas, em alguns casos insetos daninhos devido ao hábito de cortar flores, folhas e cortiça com as mandíbulas, para conseguir material necessário para a construção dos ninhos, ou para penetrar nos nectários de algumas flores prejudicando sua floração (Nogueira-Neto 1962). Por outro lado, em vários casos essas abelhas podem ser consideradas como úteis polinizadoras do Allium sativum Linnaeus (alho), Allium cepa Linnaeus (cebola), Brassica oleracea Linnaeus (couve-flor), em sua maioria possíveis de serem cultivadas em estufa (Costa \& Amaral 1976, Pirani \& Cortopassi-Laurino 1994).

De acordo com Harvey \& Brown (1951), o consumo de oxigênio é uma expressão quantitativa das atividades de um organismo intacto, podendo por sua vez, ser utilizado como uma forma de avaliação do desempenho fisiológico. Portanto, o metabolismo respiratório seria uma resposta imediata que repre- sentaria o tempo de ação de um fator sobre um organismo.

A influência da temperatura no metabolismo respiratório de insetos pode ser explorada través de experimentos com respirometria e geralmente é expressa por uma curva Respiração-Temperatura (R-T), onde é comum encontrar faixas de temperatura em que a respiração não sofre muita alteração. Essas zonas de "platô" segundo Precht et al. (1973) coincidem com temperaturas biologicamente importantes para o animal, representando zonas de adaptação térmica.

STussi \& HAMERLin (1966) estudaram o consumo de oxigênio de operárias isoladas de Apis mellifera mellifera (Linnaeus, 1758) no verão e no inverno submetidas a diferentes temperaturas e registraram que as taxas respiratórias foram mais elevadas no verão do que no inverno.

Shuel \& Dixon (1968) determinaram a influência da sazonalidade e do ambiente externo na taxa de consumo de oxigênio em larvas de rainhas de A. mellifera mellifera (72 a 96 horas de idade) comprovando também que as taxas respiratórias foram muito mais elevadas no verão do que no inverno. Verma \& Emards (1971) estudaram na Índia, a aclimatização 
metabólica e a tolerância à variações da temperatura em $A$. mellifera e A. cerana indica (Fabricius, 1798) e verificaram que as taxas respiratórias das duas espécies aumentaram regularmente com a temperatura.

CAHILl \& Lustick (1976) estudaram o consumo de oxigênio de zangões de $A$. mellifera e observaram que as taxas respiratórias foram inversamente proporcionais às temperaturas ambiente. Entretanto, as taxas respiratórias médias de zangões não apresentaram essa relação inversa e variaram muito nas baixas temperaturas.

FAHRENHOLZ et al. (1992) realizaram investigações calorimétricas em diferentes castas de A. mellifera carnica (Pollman, 1879) sendo que, na maioria dos casos, a temperatura mostrou uma significativa influência sobre o metabolismo, embora isso não tenha acontecido de forma homogênea entre as castas. Crailsherm et al. (1999) avaliaram o consumo de oxigênio de forrageiras isoladas de A. mellifera carnica e também observaram uma maior taxa respiratória de acordo com o aumento da temperatura. LeONHARD \& CRAILSHeim (1999) trabalhando também com $A$. mellifera carnica observaram que essas abelhas são ativas em ambientes com temperaturas entre 10 e $45^{\circ} \mathrm{C}$ sendo que a $51^{\circ} \mathrm{C}$ a taxa de consumo de oxigênio caiu abaixo da curva obtida para baixas temperaturas.

STABENTHEInER et al. (2003) mediram comparativamente o consumo de oxigênio entre forrageiras e operárias internas de meia-idade de $A$. mellifera mellifera e comprovaram que a taxa respiratória dependeu amplamente da variação crescente da temperatura, tendo sido observado um maior consumo de oxigênio por parte das abelhas forrageiras.

Com relação aos meliponíneos, apesar das características básicas de sua biologia indicarem um bom controle da temperatura dos ninhos, ainda são raros os trabalhos relacionando temperatura e metabolismo respiratório.

Proni \& Hebling (1996) observaram um aumento nas taxas respiratórias juntamente com a elevação da temperatura em Tetragonisca angustula fiebrigi (Schwarz, 1938) e T. angustula angustula (Latreille, 1807). Além disso foi observado que, de modo geral, as taxas respiratórias foram mais elevadas no verão do que no inverno.

Os objetivos do presente trabalho foram os de fornecer dados para o entendimento de possíveis adaptações às mudanças de temperatura no meio ambiente e também determinar suas preferências térmicas que poderão indicar com mais exatidão a distribuição geográfica natural dessas abelhas.

\section{MATERIAL E MÉTODOS}

A termogênese é conseqüência direta do metabolismo energético de um organismo e, o método mais utilizado para sua determinação é através da obtenção da taxa de produção de dióxido de carbono expelido e/ou de consumo de oxigênio. A razão entre essas taxas, conhecida como quociente respiratório $(\mathrm{QR})$, fornece ainda uma estimativa da mistura de carbohidratos, proteínas e lipídeos que estão sendo metabolizados e o equivalente calórico do oxigênio consumido. Para animais com metabolismo aeróbio, normalmente admite-se um QR de 0,8 como padrão e que corresponde a 4,8 calorias para cada centímetro cúbico de oxigênio consumido. Portanto, multiplicando-se o consumo de oxigênio por unidade de tempo, por esse valor calórico de oxigênio, pode-se expressar o metabolismo energético em termos de caloria liberada por unidade de tempo. Quando esse produto é dividido pela massa corpórea do animal, obtém-se a taxa respiratória. Sobre as relações metabólicas dos animais, são definidos três níveis: metabolismo padrão, metabolismo de rotina e metabolismo ativo. O primeiro tipo corresponde ao metabolismo basal ou de atividade mínima do animal; o segundo refere-se a um padrão em que os animais têm movimentos restritos mas não completamente imobilizados; o terceiro nível refere-se ao metabolismo ativo e está relacionado com uma atividade máxima, como por exemplo o vôo (Prosser 1968).

No presente trabalho foi posto em prática o segundo tipo, ou seja, o metabolismo respiratório de rotina através de medidas de taxas respiratórias do oxigênio consumido de abelhas forrageiras isoladas em frascos que não imobilizaram, apenas restringiam o vôo.

O experimentos com metabolismo respiratório foram realizados em respirômetro de Warburg com banho termostatizado nas temperaturas de $5 ; 10 ; 15 ; 20 ; 25 ; 30$ e $35^{\circ} \mathrm{C}$ e os frascos utilizados tiveram volumes variando de 17.1616 a 18.4449 $\mathrm{ml}$. Foram feitas determinações do consumo de oxigênio de abelhas forrageiras isoladas nos meses de outubro de 2002 a fevereiro de 2003 e nos meses de junho a agosto de 2004, sempre com exemplares do mesmo ninho localizado no meliponário da Universidade Estadual de Londrina. As temperaturas médias da região variam entre 18 e $28^{\circ} \mathrm{C}$ durante o verão e entre 12 e $23^{\circ} \mathrm{C}$ no inverno (Simepar).

As abelhas foram coletadas na entrada do ninho e transportadas para uma câmara climática com temperatura controlada a $25^{\circ} \mathrm{C}$. Para a realização dos experimentos os insetos foram colocados, individualmente, em cada frasco do respirômetro. Em cada experimento foram utilizados cinco frascos contendo abelhas e um frasco sem o inseto que funcionou como termobarômetro (TB). No poço central de cada frasco foi colocada uma fita de papel de filtro e $0,3 \mathrm{ml}$ de $\mathrm{KOH}$ a $14 \%$, para a absorção do $\mathrm{CO}_{2}$ eliminado na respiração.

Os conjuntos formados por frascos e manômetros foram levados ao banho termostatizado do respirômetro e, esperou-se 15 minutos com todo o sistema aberto, para fins de estabilização. Após esse procedimento, os níveis manométricos foram ajustados a $150 \mathrm{~mm}$ (nível inicial padrão) e os manômetros foram fechados para a tomada das leituras iniciais. Em segui$\mathrm{da}$, foram feitas leituras em intervalos de 15 minutos, durante 75 minutos, desprezando-se, no cômputo do consumo de oxigênio, os 15 minutos iniciais do experimento.

Ao final de cada experimento as abelhas foram removidas para serem deterninados o peso e o volume. De posse des- 
ses dados calculou-se a constante do conjunto (frasco e manômetro) de acordo com a metodologia citada por UMBReIT et al. (1972).

Com a constante de cada conjunto calculou-se o consumo de oxigênio, multiplicando-se a mesma pela soma das variações manométricas (h). Dividindo-se o oxigênio consumido durante 60 minutos pela massa corpórea de cada abelha (em $\mathrm{mg}$ ), obteve-se o valor de $\mathrm{VO}_{2}$ (taxa respiratória) em $\mathrm{ml} \mathrm{O}_{2} / \mathrm{mg}$ /hora. Os dados obtidos foram analisados através de Anova Fatorial e posteriormente submetidos ao teste de Tukey (1953).

Para uma análise estatística das taxas respiratórias de $T$. spinipes em relação às temperaturas e estações do ano, os dados foram transformados para $\log (\mathrm{x}+1)$, num experimento conduzido segundo o delineamento fatorial $(2 \times 7)$, inteiramente ao acaso, com 10 repetições. Os fatores analisados foram: 1) estações do ano em dois níveis (verão e inverno) e; 2) temperatura em sete níveis $\left(5,10,15,20,25,30\right.$ e $\left.35^{\circ} \mathrm{C}\right)$.

\section{RESULTADOS}

As taxas respiratórias médias de dez abelhas forrageiras de $T$. spinipes, obtidas em várias temperaturas, nos períodos de verão e inverno estão na figura 1. A análise estatística dos dados mostrou que a taxa respiratória aumentou diretamente com a elevação da temperatura em ambos os períodos. Analisando-se os dados em relação à sazonalidade, observou-se um maior consumo de oxigênio durante os meses de inverno, principalmente a partir de $20^{\circ} \mathrm{C}$.

A análise estatística das taxas respiratórias de $T$. spinipes em relação às temperaturas e estações do ano para os dados transformados podem ser observados na tabela I.

O teste $\mathrm{F}$ mostrou que os efeitos da sazonalidade temperatura e da interação entre estação e temperatura foram significativos para $\mathrm{p}<0,0051$. Dada a significância da interação entre a estação do ano e a temperatura, foi aplicado o teste de TuKEY ao nível de significância de 5\% para comparar as médias dos dados transformados, duas a duas, para avaliar o efeito das estações do ano em cada temperatura e, das temperaturas em cada estação.

Os resultados apresentados na tabela II mostram que as médias das estações verão e inverno em cada temperatura diferem significativamente a $15 ; 20$ e $35^{\circ} \mathrm{C}$. A média da $\mathrm{VO}_{2}$ foi menor no inverno do que no verão para $15^{\circ} \mathrm{C}$ e maior nas temperaturas de 20 e $35^{\circ} \mathrm{C}$.

No inverno, foi verificado que a média da $\mathrm{VO}_{2}$ a $35^{\circ} \mathrm{C}$ foi significativamente maior do que nas demais temperaturas e, as temperaturas $20 ; 25$ e $30^{\circ} \mathrm{C}$ não apresentaram diferenças significativas constituindo-se praticamente num platô, porém diferindo das temperaturas $5 ; 10$ e $15^{\circ} \mathrm{C}$ que apresentaram médias menores de $\mathrm{VO}_{2}$.

Durante o verão, houve uma mudança significativa nas relações entre taxas respiratórias e temperaturas pois, a média da $\mathrm{VO}_{2}$ na temperatura de $35^{\circ} \mathrm{C}$ não apresentou diferença significativa das temperaturas 30 e $25^{\circ} \mathrm{C}$ (platô entre 35 e $25^{\circ} \mathrm{C}$ e

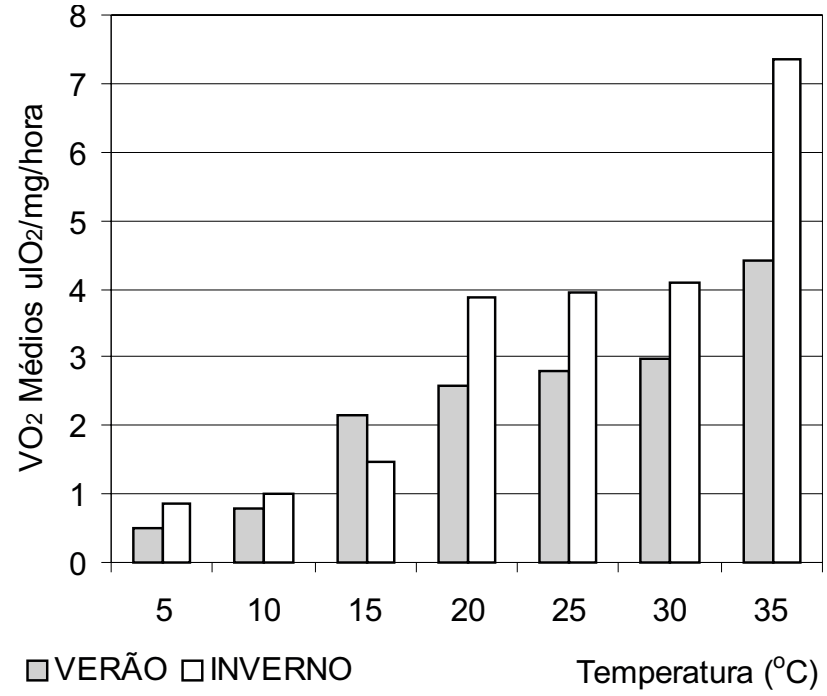

Figura 1. Taxas respiratórias médias de abelhas forrageiras $T$. spinipes em diferentes temperaturas no verão e inverno.

entre 30 e $15^{\circ} \mathrm{C}$ ). Além disso verificou-se que 5 e $10^{\circ} \mathrm{C}$ foram as temperaturas onde foram obtidas as menores médias da $\mathrm{VO}_{2}$, diferindo significativamente das demais.

\section{DISCUSSÃO}

Os dados obtidos mostraram que, isoladamente essas abelhas apresentaram um comportamento típico de animais poiquilotermos, ou seja, elevaram a taxa respiratória com o aumento da temperatura, principalmente nos extremos da curva respiração-temperatura. Com relação aos efeitos da sazonalidade, os resultados mostraram um consumo maior de oxigênio nos meses de inverno em comparação com o consumo nos meses de verão nas faixas mais altas de temperatura $\left(20\right.$ a $\left.30^{\circ} \mathrm{C}\right)$, embora o inverso tenha ocorrido a $15^{\circ} \mathrm{C}$.

A análise de variância da comparação entre os fatores estações do ano e as taxas respiratórias mostraram uma diferença significativa entre as temperaturas $15 ; 20$ e. $35^{\circ} \mathrm{C}$ no verão e inverno. Para essas temperaturas, as médias de $\mathrm{VO}_{2}$ foram maiores no inverno do que no verão. No inverno verificou-se que a média da taxa respiratória na temperatura de $35^{\circ} \mathrm{C}$, foi maior do que a média obtida a $35^{\circ} \mathrm{C}$ no verão, mostrando que elevações repentinas de temperatura durante o inverno podem ocasionar elevações mais acentuadas no metabolismo respiratório.

Heath (1971) afirmou que os insetos, representando o grupo mais abundante e diverso dos organismos terrestres, tendem a ser sazonais e dessa forma, limitados à faixas de temperatura ambiente que devem enfrentar com seus estágios mais ativos. Assim, seletividade termal e sazonal seriam adaptações primárias dos insetos para a existência terrestre.

Com base em diferenças nas temperaturas de coma, taxas respiratórias e atividades enzimáticas existem evidências 
Tabela I. Análise de variância entre estações do ano e temperaturas para abelhas forrageiras de T. spinipes. (G.L.) Grau de liberdade, (S.Q.) soma de quadros, (Q.M.) quadrado médio.

\begin{tabular}{lrrrrr}
\hline \multicolumn{1}{c}{ Causas da variação } & G.L & S.Q. & Q.M. & Valor de F & Prob. > F \\
\hline Estação & 1 & 0,5105 & 0,5105 & 8,5628 * & 0,00441 \\
Temperatura & 6 & 31,7157 & 5,2859 & 88,3491 & 0,00001 \\
Estação x Temperatura & 6 & 1,9372 & 0,3229 & 5,3963 * & 0,00015 \\
Resíduo & 126 & 7,5386 & 0,0598 & - \\
\hline Total & 139 & 41,7020 & - & - \\
\hline
\end{tabular}

* $<0,05$ (valor de F); média geral: 1,1503; coeficiente de variação: 21,264 \%.

Tabela II. Teste de Tukey para a análise dos efeitos da interação estações do ano e temperaturas para abelhas forrageiras T. spinipes.

\begin{tabular}{cll}
\hline Temperatura $\left(^{*}\right)$ & Verão $\left(^{* *}\right)$ & Inverno $\left(^{* *}\right)$ \\
\hline 5 & 0,717 a C & 0,4468 a C \\
10 & 0,766 a C & 0,700 a C \\
15 & 1,301 a C & 2,691 b B \\
20 & 3,448 a B & 2,384 b B \\
25 & 3,493 a B & 2,789 a AB \\
30 & 3,775 a B & 3,131 a AB \\
35 & 7,294 a A & 4,247 b A \\
\hline
\end{tabular}

*) médias de temperaturas seguidas por letras maiúsculas distintas nas colunas diferem entre si ao nível de significância de $5 \%$ (d.m.s. $=0,32764$ para dados transformados em $\left.\log (x+1) ;{ }^{* *}\right)$ médias de estação seguidas por letras distintas minúsculas e nas linhas, diferem entre si a nível de significância de $5 \%$ (d.m.s. = 0,21644 para dados transformados em $\log (x+1)$.

que os insetos mostram duas diferenças básicas nas suas respostas às variações da temperatura: interespecífica (adaptativa) e intraespecífica (aclimativa). As diferenças adaptativas tem sido correlacionadas com temperaturas do ambiente normal do inseto e as diferenças aclimativas com a história termal do inseto (Anderson \& Mutchmor 1971).

PRECHT et al. (1973) estabeleceram uma diferença entre os termos aclimação e aclimatização. Assim, aclimação deve ser utilizada quando a adaptação de um organismo envolve apenas um fator, como ocorre em experimentos controlados em laboratório. Aclimatização é usada quando envolve um complexo de fatores do ambiente, tais como, mudanças sazonais ou climáticas.

$\mathrm{Na}$ literatura pode-se observar que a maioria dos dados obtidos sobre os efeitos da temperatura no metabolismo respiratório de abelhas mostraram uma relação direta entre taxa respiratória e aumento da temperatura. Registros antigos como os de SAyle (1928) e Allem (1959) concluíram que, de modo geral, o consumo de oxigênio de abelhas do gênero Apis aumentava com a elevação da temperatura.

Verma \& EDWARDS (1971) observaram que as taxas respiratórias de A. mellifera mellifera e A. cerana indica aumentaram regularmente com o aumento da temperatura, exceto no outono, onde as curvas mostraram um pico a $30^{\circ} \mathrm{C}$. As comparações dessas curvas de outono e inverno mostrou evidência de aclimatização térmica sazonal em ambas as espécies sugerindo assim uma aclimatização sazonal. SHUEel \& DiXON (1968) estudaram a respiração de $A$. mellifera mellifera durante a fase larval observando que as taxas respiratórias de larvas de rainha foram também muito mais elevadas no verão do que no outono, embora essas variações tenham sido bem menos acentuadas em larvas de operárias.

Estudos posteriores com investigações calorimétricas desenvolvidos por FAHRENHOLz et al. (1992) chegaram às mesmas conclusões. Trabalhando com diferentes castas de A. mellifera carnica, na maioria dos casos, a temperatura mostrou uma influência significativa sobre o nível metabólico, embora isso não tenha ocorrido de forma uniforme entre as castas. CRAILSHEIm et al. (1999) compararam o consumo de oxigênio de forrageiras isoladas de A. mellifera carnica a 25 e $35^{\circ} \mathrm{C}$ em respirômetro de Warburg. Os resultados vão de encontro aos anteriores mostrando uma diferença significativa na taxa respiratória diretamente ligada com o aumento da temperatura.

Com relação aos meliponídeos, Proni \& Hebling (1996) observaram um aumento das taxas respiratórias com a elevação da temperatura em Tetragonisca angustula fiebrigi e $T$. angustula angustula. Foram registradas as faixas onde o metabolismo estaria melhor adaptado durante o verão e inverno. Para T. angustula fiebrigi foi entre 15 e $25^{\circ} \mathrm{C}$ no inverno e entre 25 e $30^{\circ} \mathrm{C}$ no verão. T. angustula angustula, mostrou uma melhor adaptação entre 30 e $40^{\circ} \mathrm{C}$ no verão e entre 25 e $30^{\circ} \mathrm{C}$ no inverno. Além disso foi observado que, de modo geral, as taxas respiratórias foram mais elevadas no verão do que no inverno.

Nos experimentos realizados com $T$. spinipes, a taxa de consumo de oxigênio aumentou de acordo com o aumento da temperatura dos experimentos e também foi mais elevada no inverno do que no verão, o que difere da maioria dos trabalhos relacionados anteriormente. Durante os meses de verão foi observado um aumento irregular no consumo de oxigênio na temperatura de $15^{\circ} \mathrm{C}$ indicando uma dificuldade de adaptação diante de uma possível queda repentina da temperatura durante o verão. Com relação às preferências térmicas observou-se tanto no inverno como no verão um intervalo entre 20 e $30^{\circ} \mathrm{C}$, 
onde o consumo de oxigênio se estabilizou mostrando uma possível faixa térmica adaptativa ideal para as abelhas forrageiras de T. spinipes.

\section{AGRADECIMENTO}

À Profa Dra Tiemi Matsuo pelo auxílio na análise estatística.

\section{REFERÊNCIAS BIBLIOGRÁFICAS}

Anderson, R.L. \& J.A. Mutchmor 1971. Temperature acclimation in Tribolium and Musca at metabolic and enzyme levels. Journal of Insect Physiology, Oxford, 17: 2205-2219.

AlLEN, M.D. 1959. Respiration rates of worker honeybees of different ages and different temperatures. Journal of Experimental Biology, Cambridge, 36 (1): 92-101.

Cahill, K. \& S. Lustick 1976. Oxygen consumption and thermoregulation in Apis mellifera workers and drones. Comparative Biochemistry and Physiology, Vancouver, 55 (A): 355-357.

Costa, J.D. \& E. Amaral. 1976. Produção de sementes de Crotalaria juncea L. na presença e na ausência de insetos polinizadores. Revista de Agricultura, Havana, 51: 57-60.

Crailsheim,K; A. Stabentheiner; N. Hrassnigg \& B. Leonhard 1999. Oxygen consumption at different activity levels and ambient temperatures in isolated honeybees (Hymenoptera: Apidae). Entomologia Generalis, Stuttgart, 24 (1): 1-12

Fahrenholz, L.; I. Lamprecht \& B. Schircker. 1992. Calorimetric investigations of the energy metabolism of the honeybee workers Apis mellifera carnica. Journal of Comparative Physiology, Berlin, 162: 119-130.

Harvey, G.T. \& A.W.A. Brown. 1951 The effect of insecticides on the rate of oxygen consumption in Blattella. Canadian Journal of Zoology, Montreal, 29: 42-53.

Неатн, J.E. 1971. Adaptation of the thermal responses of insects. American Zoologist, Seattle, 11: 146-156.

Leonhard, B; B. Crailsheim 1999. Temperature dependency of the oxygen consumption by a thorax homogenate of worker honeybees (Hymenoptera: Apidae). Entomologia Generalis, Stuttgart, 24 (1): 31-36.
Nogueira-Neto, P. 1962. The scutelum nest structure of Trigona (Trigona) spinipes (Fabr., 1793) (Hymenoptera: Apidae). Journal of New York Entomological Society, New York, 70: 239-264.

Pirani, J.R. \& M. Cortopassi-Laurino. 1994. Flores e abelhas em São Paulo. São Paulo, Edusp, 192p.

Precht, H.; J. Christophers \& H. Hensel. 1973. Temperature and life. New York, Springer Verlag, 779p.

Proni, E.A. \& M.J.A. Hebling. 1996. Thermoregulation and respiratory metabolism in two brazilian stingless bee subspecies of different climatic distribution, Tetragonisca angustula fiebrigi and T. a. angustula (Hymenoptera: Apidae: Meliponinae). Entomologia Generalis, Stuttgart, 20 (4): 281-289.

Prosser, C.L. 1968. Temperatura, p 256-306. In: C.L. Prosser \& F.A. Brown Jr. (Eds). Fisiologia Comparada. México, Editora Interamericana, $2^{\text {a }}$ ed., $966 \mathrm{p}$.

SAYLE, M.H. 1928. The metabolism of insects. Quarterly Review of Biology, Chicago, 3 (4): 542-553.

Shuel, R.W. \& S.E. Dixon 1968. Respiration in developing honeybee larvae. Journal of Apicultural Resesearch, Cardif, 7 (1): 11-19.

Stabentheiner, A.; J. Vollman; H. Kovac \& K. Crailshein. 2003. Oxygen consumption and body temperature of active and resting honeybees. Journal of Insect Physiology, Oxford, 49 (9): 881-889.

Stussi, T. \& M.L. Harmelim. 1966 Evolution saisonnière de la thermogenese de l'abeille. Étude de la consomation d'oxygène de l'abeille isolée en été et en hiver à cinq températures differentes. Comptes Rendus de la Societé du Biologie. Paris, 160: 503-1506.

Tukey, J.W. 1953. The problem of multiple comparisons. Princeton, Princeton University, IX+189p.

Umbreit, W.W.; R.H. Burris \& J.F. Stauffer. 1972. Manometric biochemical techniques. Minneapolis, Burges Publications Company, $5^{\text {th }}$ ed., $387 p$.

Verma, L.R. \& D.K. EDWARDS. 1971. Metabolic aclimatization to temperature and temperature tolerance in Apis mellifera and A. cerana indica kept in India. Journal of Apicultural Research, Cardiff, 10 (3): 105-108.

Recebido em 06.IV.2005; aceito em 23.XI.2005. 